\title{
Controllable dispersion and reunion of liquid metal droplets
}

\author{
Sen Chen ${ }^{1,2}$, Yujie Ding ${ }^{1,2}$, Qinglei Zhang ${ }^{1,2}$, Lei Wang ${ }^{1 *}$ and Jing Liu ${ }^{1,2,3^{*}}$
}

\begin{abstract}
Liquid metal (LM) micro-droplets have been widely used in microfluidics, drug-loaded nano-system and micro-nano machine due to its excellent properties. However, there still exist difficulties in succinctly dispersing a bulk of LM into micro-droplets due to the large interfacial tension. Besides, the controllable switching between droplet dispersion and reunion is yet to be realized. Herein, a practical and efficient method for dispersing LM was proposed and the controllable switching between dispersion and reunion of LM droplets was achieved. LM micro-droplets were produced by vibrating the LM immersed in a mixture of $\mathrm{N}, \mathrm{N}$-dimethylformamide (DMF) and polyvinyl chloride (PVC). The experimental results show that the size distribution of $\mathrm{LM}$ micro-droplets could be tuned by controlling the vibration frequency. More intriguingly, the dispersion and reunion of LM droplets can be switched intelligently through tuning the vibration frequency and amplitude. Furthermore, optical properties of the LM micro-droplet coating were evaluated to display potential applications. A self-driven motion of PVCcoated LM could be achieved by utilizing the produced LM micro-droplets based on the Marangoni effect, which holds promising value for developing future transport tool of LM droplets. The present work suggests an entirely feasible method for dispersing and utilizing LM droplets, which is of great significance for promoting the development of LM micro-droplet science and technology.
\end{abstract}

Keywords: liquid metal, micro-droplet, dispersion and reunion, self-driven motion, optical property

\section{INTRODUCTION}

Room temperature liquid metals (LMs) represented by Ga-based alloys, such as EGaIn $\left(\mathrm{Ga}_{75.5} \mathrm{In}_{24.5}\right)$ and Galinstan $\left(\mathrm{Ga}_{68.5} \mathrm{In}_{21.5} \mathrm{Sn}_{10}\right)$, feature many useful properties including low melting point, high electrical and thermal con- ductivity, large interfacial tension, negligible vapor pressure and low viscosity [1-3]. Therefore, they have attracted intense attention in the areas of flexible electronics and sensors [4-7], thermal management [8-10], $3 \mathrm{D}$ printing [11,12], microfluidics [13-15], drug-loaded nano-systems and micro-nano machines [16-18]. With the wide application and growing demand of LM microdroplets, the method for dispersing LM into micro-droplets has gained much attention and got some progress.

Several methods have been developed for producing LM micro-droplets including electric field [19-21], sonication [22,23], pneumatic dispersion [24] and flow-focusing $[25,26]$, etc. However, there exist disadvantages more or less among them. For example, the dispersed LM micro-droplets are easily reunited when they contact each other in the case of electric field. Besides, some methods are limited to producing large sphere diameters $(\geq 100 \mu \mathrm{m})$ and the mass production of such micro-droplets would be time-consuming (sonication). As for the method of microfluidic flow-focusing and pneumatic dispersion, the system for producing micro-droplets is usually rather complex and huge, which is not portable and versatile. Therefore, succinctly dispersing LM into micro-droplets with portable equipment and practicable method plays a crucial role in the application of LM micro-droplets. On the other hand, previous studies focused more on how to disperse LM than the reunion of LM droplets. There is no doubt that the switch between dispersion and reunion of LM is also important for many applications.

In this work, a simple and efficient method for producing the micro-droplets with controlled size was proposed. A large number of LM micro-droplets were generated by vibrating the bulk LM in a mixed solution of $\mathrm{N}, \mathrm{N}$-dimethylformamide (DMF) and polyvinyl chloride

\footnotetext{
${ }^{1}$ Technical Institute of Physics and Chemistry, Chinese Academy of Sciences, Beijing 100190, China

${ }^{2}$ School of Future Technology, University of Chinese Academy of Sciences, Beijing 100049, China

${ }^{3}$ Department of Biomedical Engineering, School of Medicine, Tsinghua University, Beijing 100084, China

*Corresponding authors (emails: jliu@mail.ipc.ac.cn (Liu J); leiwang@mail.ipc.ac.cn (Wang L))
} 
a $f=0.1-20 \mathrm{~Hz} ; A=20 \mathrm{~cm}$
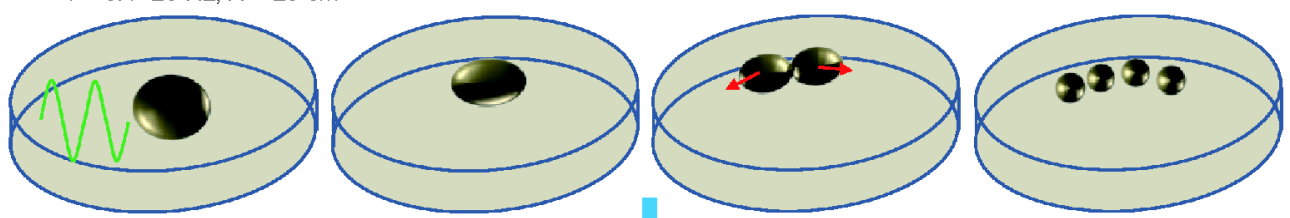

b $f=1-50 \mathrm{kHz} ; A=0.01 \mathrm{~cm}$
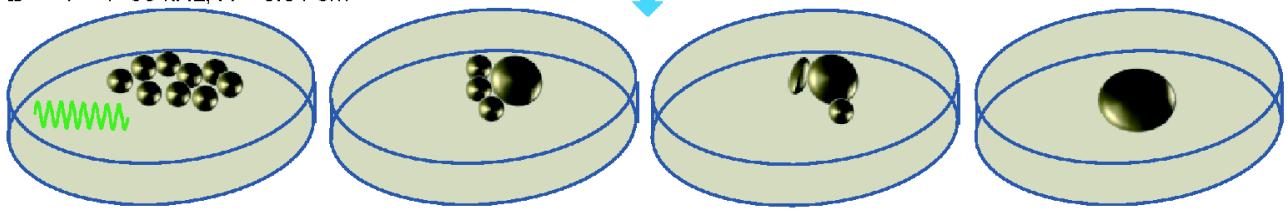

c
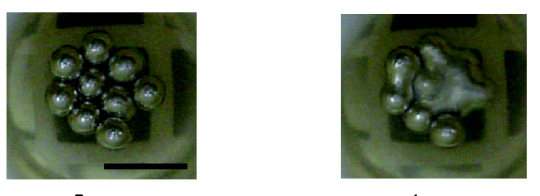

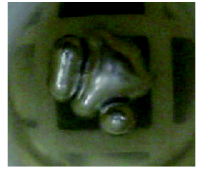

$4 \mathrm{~ms}$

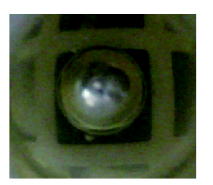

$7 \mathrm{~ms}$

Figure 1 (a) The dispersion of LM droplets occurs under the condition of low frequency and high amplitude of vibration. (b) The reunion of LM droplets occurs under the condition of high frequency and low amplitude of vibration. (c) Time-lapse images of the reunion of LM droplets. The scale bar is $10 \mathrm{~mm}$.

(PVC) and the controlled size distribution was realized by tuning the vibration frequency. Besides, controllable switching between the dispersion and the reunion of LM droplets was achieved by controlling the vibration frequency and amplitude. Such controllable switching between dispersion and reunion of LM droplets is expected to be applied in many potential areas, such as deformable soft machine. Based on the LM micro-droplets produced in this method, two potential applications of LM microdroplets were demonstrated. LM coating, which was manufactured by utilizing the LM micro-droplets, shows excellent optical properties. Besides, the self-driven motion of the PVC-coated LM droplets was successfully implemented, which opens up a new strategy for transporting LM droplets.

\section{EXPERIMENTAL SECTION}

Over the current experiments, the LM used was $\mathrm{Ga}_{67} \mathrm{In}_{21} \mathrm{Sn}_{12}$, which was prepared by stirring and heating gallium $(\mathrm{Ga}$, $67 \mathrm{wt} \%, 99.99 \%$ purity), indium (In, 21 wt\%, 99.99\% purity) and tin (Sn, $12 \mathrm{wt} \%, 99.99 \%$ purity) together at $150^{\circ} \mathrm{C}$ for $2 \mathrm{~h}$ until metal alloy was formed. The solution was prepared by dissolving 2 g PVC (average polymerization degree was around 300) powder in $50 \mathrm{~mL}$ DMF solution. DMF and PVC were purchased from Beijing Lan Yi Chemical Products Co., Ltd. (Beijing, China). For the experiment of the self-driven motion of PVC-coated LM droplets, the produced LM droplets were dripped on water surface in a glass petri dish, and the imaging data and the infrared images of the self-driven motion were recorded by the Sony Digital Video (FDRAX40) and the far-infrared thermograph imaging system (FLIR SC620, FLIR Systems Inc, USA), respectively. The micromorphology of the LM micro-droplets was observed by means of scanning electron microscopy (SEM; S-4800, Hitachi, Ltd.). The absorbance and the reflectivity of LM micro-droplets were measured by UV-visible nearinfrared spectrophotometer (Cary 7000).

\section{RESULTS AND DISCUSSION}

DMF is a common solvent with high boiling point, low freezing point, excellent chemical stability and thermal stability. Besides, it was proven to have extraordinary effect in dispersing a bulk of LM. Here, PVC was added to DMF for adjusting the viscosity of DMF to achieve better dispersion. Therefore, a bulk of LM can be broken into micro-droplets by vibrating the LM immersed in the PVC modified DMF solution in a beaker.

Distinctive from the traditional method, the PVC modified DMF solvent can achieve not only droplet dispersion, but also droplet reunion (Fig. 1). The key factor behind is the vibration frequency and amplitude. The dispersion of LM droplets occurred under low frequency and high amplitude of vibration. Conversely, the reunion of LM droplets took place under high frequency and low amplitude (Fig. 1a and b), which only took milliseconds 


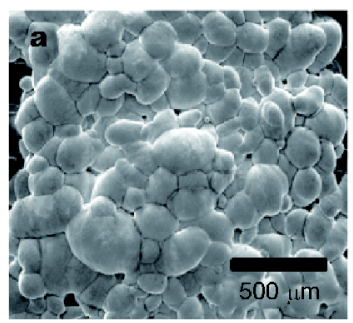

$\mathrm{AD}=200 \mu \mathrm{m}$

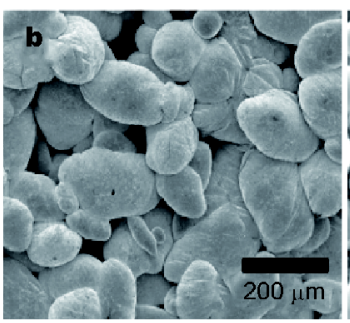

$\mathrm{AD}=80 \mu \mathrm{m}$

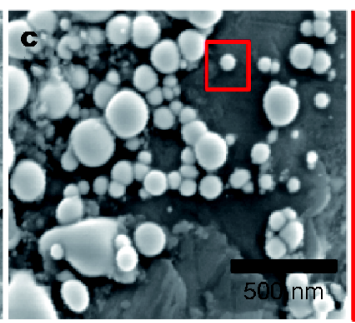

$\mathrm{AD}=60 \mathrm{~nm}$

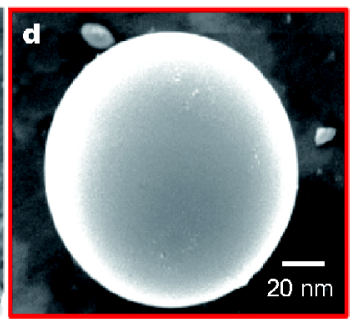

e

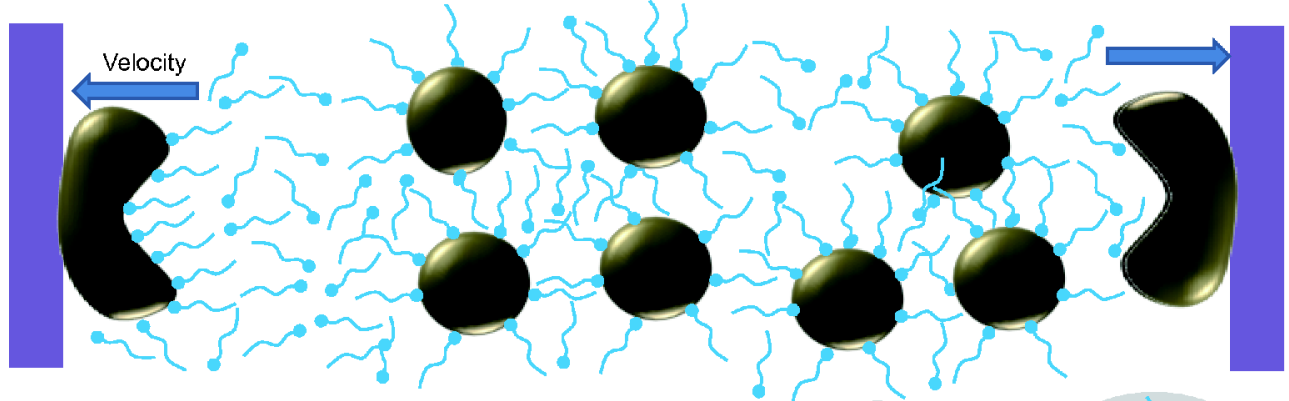

f
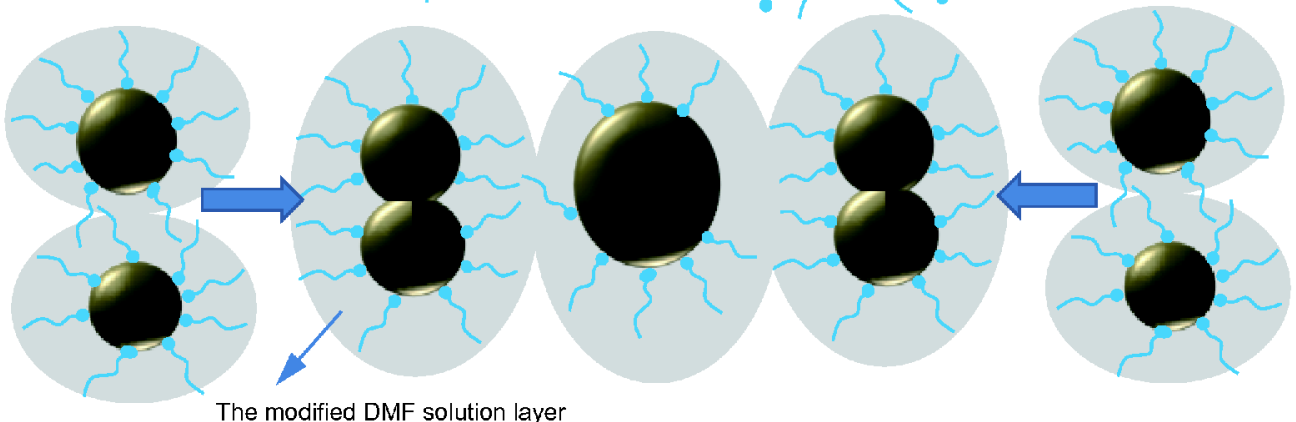

The modified DMF solution layer

Figure 2 SEM images of micro-droplets with different average diameters (AD): (a) 200, (b) 80, (c) 60, (d) 20 $\mu$ m. (e) Schematic diagram of the mechanism lying behind the dispersion. (f) Schematic diagram of the mechanism lying behind reunion of LM micro-droplets.

(Fig. 1c). More importantly, the dispersed droplets can remain stable for a long time $(>50 \mathrm{~h})$, unless high frequency and low amplitude vibrations are exerted on it, which shows the reliability of the method for dispersing LM. Such features are expected to be applied in many potential areas, such as deformable soft machine. Facing the narrow passage, LM soft machine can disperse into numerous micro-droplets to pass the narrow passage and then reunite into a complete LM soft machine.

Experimental results show that the dispersed LM droplets gradually decrease in size as the vibration time increases and the LM droplets can be dispersed into $100 \mathrm{~nm}$ eventually (Fig. 2a-d). The dispersed micro-droplets do not reunite under this condition because they are well separated by the PVC modified DMF solvent. Here, the organic solvent acting as surfactant plays a crucial role. Since the LM droplets possess a large specific surface area and a high surface energy, they are easily agglomerated. In practical applications, surfactants are usually added as dispersants to change the surface properties of the microdroplets for improving their dispersion. Here, the modified DMF solution acting as a surfactant was adsorbed on the surface of the LM droplets and formed a steric hindrance layer, which can inhibit the reunion of LM droplets (Fig. 2e). Conversely, the LM droplets can reunite when the steric hinderance was removed due to the breaking of the modified DMF solution layer by external energy in the form of high frequency and low amplitude vibration (Fig. 2f). Besides, a low amplitude vibration avoided the collision of LM on the beaker wall, and thus the re-dispersion of LM was difficult.

The size of the micro-droplets was controlled mainly by the droplet breakage which was related to the vibration frequency and amplitude. Generally, the droplet breakage depends on the speed of LM relative to the beaker. In the case of low frequency and large amplitude, LM droplets were more likely to experience a collision on the beaker wall, resulting in the dispersion of droplets (Fig. 2e). The 

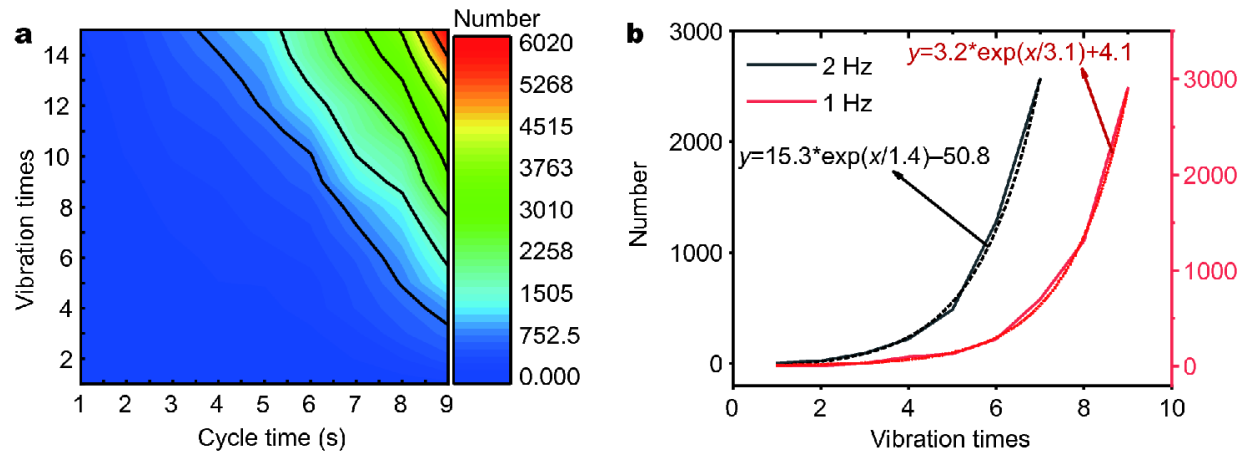

Figure 3 (a) The number of LM droplets under different vibration times and cycle time. (b) The number of LM micro-droplets increases exponentially with the number of vibrations.

collision force was taken as the main energy source. Only when the energy was large enough, could the crush of the LM droplets occur, thereby dispersing the droplets. Therefore, the ratio between the collision force and interfacial tension is related with the size of the produced LM micro-droplets.

Taking viscosity into consideration, the dimensionless number $C_{\mathrm{a}}$ can be used to describe the entire process, as expressed by the formula as follows:

$$
C_{\mathrm{a}}=\frac{\mu V}{\gamma}
$$

where $\mu$ is the viscosity of the mixed solution and $V$ is the characteristic velocity of $\mathrm{LM}$, and $\gamma$ is the interfacial tension between LM and the mixed solution. It is known that the size of micro-droplets produced varies inversely with the capillary number. Therefore, tuning the velocity and viscosity is an effective and reasonable method to control the size distribution of LM micro-droplets. In this paper, PVC was added to increase the viscosity for better dispersion. Altering the frequency and amplitude of vibration is equivalent to changing the velocity of droplets striking the beaker wall, which determines the size distribution of the droplets.

In order to better describe this phenomenon quantitatively and verify the previous analysis, we carried out an experiment to reveal the relationship between the size distribution of LM droplets and the parameters of the vibration. Experimental results show that the same volume of LM $(50 \mu \mathrm{L})$ can be dispersed into more LM micro-droplets with the increase of cycle time and vibration times (Fig. 3 and Table S1). Here, cycle time means the total time used for vibration, reflecting the adequacy of vibration. More vibration times in the same cycle time implies the larger frequencies and faster rates, resulting in the larger number of LM micro-droplets according to
Equation (1), which is consistent with the experimental results (Fig. 3a). Besides, we know that the LM will be dispersed during the process of hitting the wall of the beaker every time by the analysis above. Therefore, the number of LM micro-droplets should be exponentially related to vibration times. Based on the analysis, an experiment was carried out as shown in Fig. 3b. By fitting the curve, an exponential relationship between the number of LM micro-droplets and vibration times was confirmed, which implies the rationality of the above analysis. Therefore, the hit played a vital role here in dispersing LM droplets and a proper size distribution of LM could be controlled by the vibration frequency.

Reunion of dispersed LM micro-droplets was achieved by altering the frequency and amplitude of the vibration. The mechanism lying behind was the destruction of the surface solution layer caused by high frequency vibration. Here, the energy for removing a unit area solution layer can be represented by a parameter $a$, and the energy exerted by high frequency vibration in a unit time is thought as $b$, which is proportional to the square of vibration frequency. Hence, a formula can be derived, as shown below:

$$
N \pi D^{2} a=b t .
$$

Here $N=3 V / \pi D^{3}$, which stands for the number of dispersed LM micro-droplets. Besides, $b=A f^{2}$ ( $A$ is the proportionality constant). $V$ stands for the volume of LM and $t$ means the reunion time. Therefore, the relationship among the reunion time $(t)$, average diameter $(D)$ and frequency $(f)$ could be deduced as follows:

$$
t=\frac{3 V a}{A f^{2} D} \text {. }
$$

As a result, one can draw a conclusion that the droplet reunion time is inversely proportional to the average diameter and the square of frequency. 

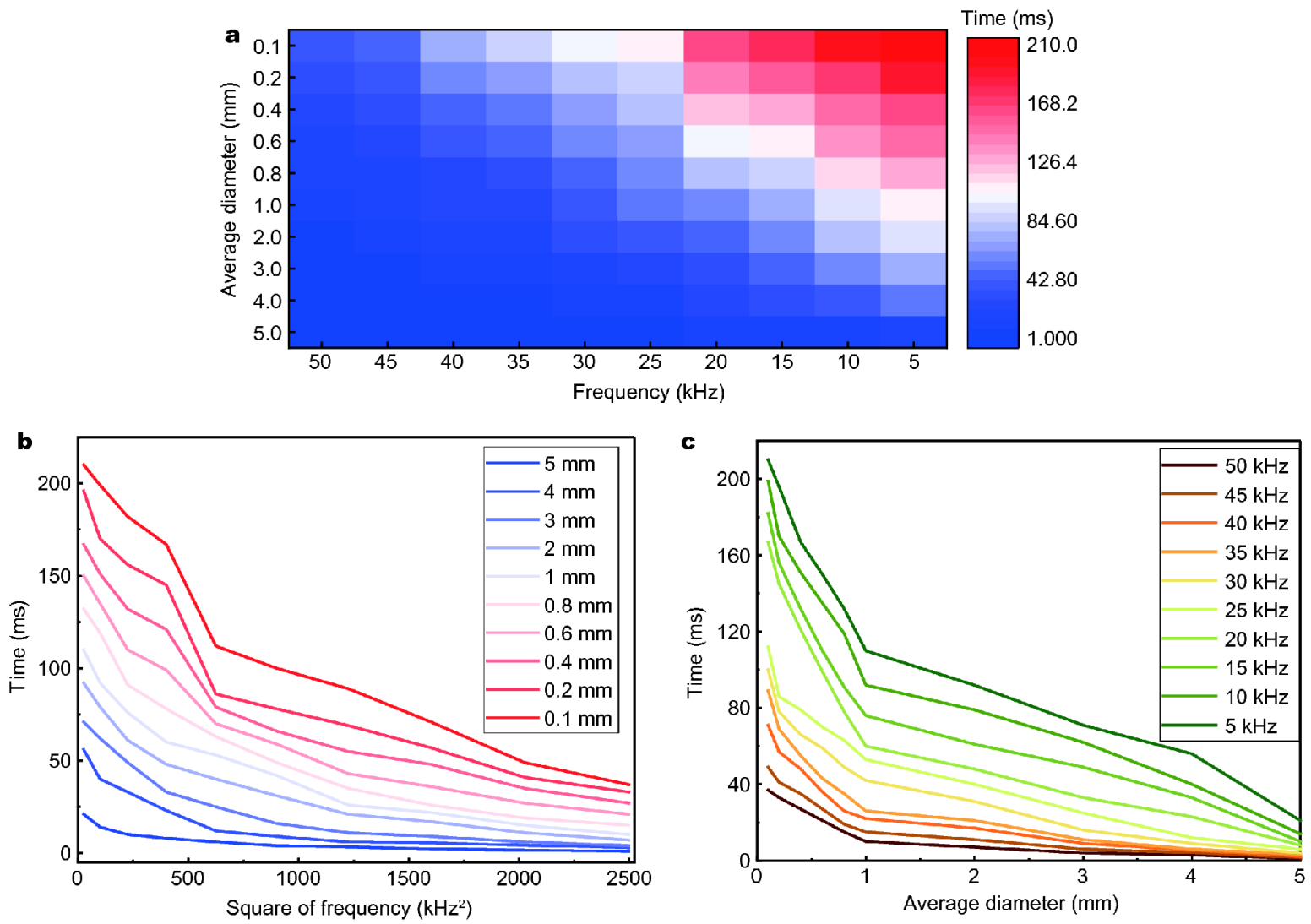

Figure 4 (a) The relationship between the reunion time and vibration frequency of LM micro-droplets with different average diameter. (b) The time of reunion is inversely proportional to the square of frequency. (c) The time of reunion is inversely proportional to the average diameter.

To verify this analysis, the experiment of LM reunion time with different average diameter in different vibration frequency was implemented (Table S2). Experimental results show that the reunion process only took milliseconds for LM micro-droplets produced by the same volume of LM (Fig. 4a), which indicates the high efficiency of the reunion of LM micro-droplets. Moreover, the reunion time is inversely proportional to the average diameter of LM droplets and the square of vibration frequency based on the theoretical analysis, which is also confirmed by our experiments. The results demonstrate that the LM droplets with large size have the fastest reunion speed under high vibration frequency (Fig. 4), thus proving that reunion efficiency is proportional to collision energy. Furthermore, Fig. $4 \mathrm{~b}$ and $\mathrm{c}$ demonstrate that the reunion time of LM micro-droplets is inversely proportional to the average diameter and the square of vibration frequency.

Hence, the reunion process of LM micro-droplets can be regulated by altering the vibration frequency and the droplet size based on the verified theoretical analysis above. The relationship between reunion speed of LM micro-droplets and vibration frequency was revealed quantitatively, providing more ways to regulate the reunion of LM droplets, which is of great significance for the potential application.

Coating with varied optical properties is widely used to modify the surface [27-29]. Here, the LM micro-droplet coating was manufactured on the surface of glass. Even if the LM coating has superb opacity and electromagnetic shielding effect, there exists a concern whether dispersed LM micro-droplets can maintain the same properties. For highlight the characteristics of LM micro-droplet coating and demonstrate its potential applications, the differences in optical properties between the LM micro-particle coating and the common LM coating were studied. As shown in Fig. 5, the absorbance of LM micro-droplets remains above three, comparable to that of the bulk LM in the visible and infrared regions, though the values are slightly lower for the LM micro-droplets. These results indicate that the LM micro-droplets still have excellent opacity and electromagnetic shielding effect to meet the 


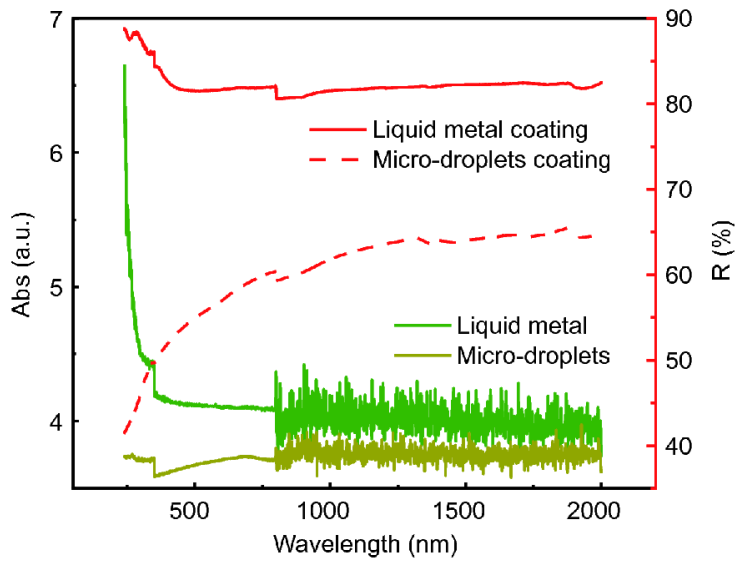

Figure 5 Comparison of absorbance (Abs) of bulk LM and LM microdroplets $(10 \mu \mathrm{m})$ placed in a $10-\mathrm{mm}$ cuvette, and the reflectivity $(R)$ of LM coating and LM micro-droplet coating is also compared as marked in red line.

requirements.

The reflectivity $(R)$ of LM coating and LM micro-droplet coating $(10 \mu \mathrm{m})$ was also tested. The results show that the reflectivity of LM micro-droplet coating is lower than that of LM coating (Fig. 5), which means that the reflectivity of the coating can be adjusted by controlling the size and proportion of the micro-droplets to meet different requirements for extensive applications. Besides, the LM micro-droplet coating consisting of PVC and LM can avoid the flow of LM due to the occurrence of liquidsolid phase transition of PVC, which is beneficial for the usage in many occasions.

On the other hand, the bulk LM was successfully dispersed into micro-droplets consisting of a LM core and a shell formed from the mixed solution (Fig. $6 a$ and $d$ ). Based on such core-shell structure that can also be seen in other studies [30], the self-driven motion of PVC-coated LM droplets was realized on water surface (Fig. 6), which is a breakthrough for the transport of LM on the water surface. The mechanism behind the self-driven movement was the Marangoni effect [31-34]. Interfacial tension gradients play an essential role in the motion.

Experimental results show that self-driven motion took place when the LM micro-droplets were placed on water surface (Movie S1). PVC-coated LM micro-droplets

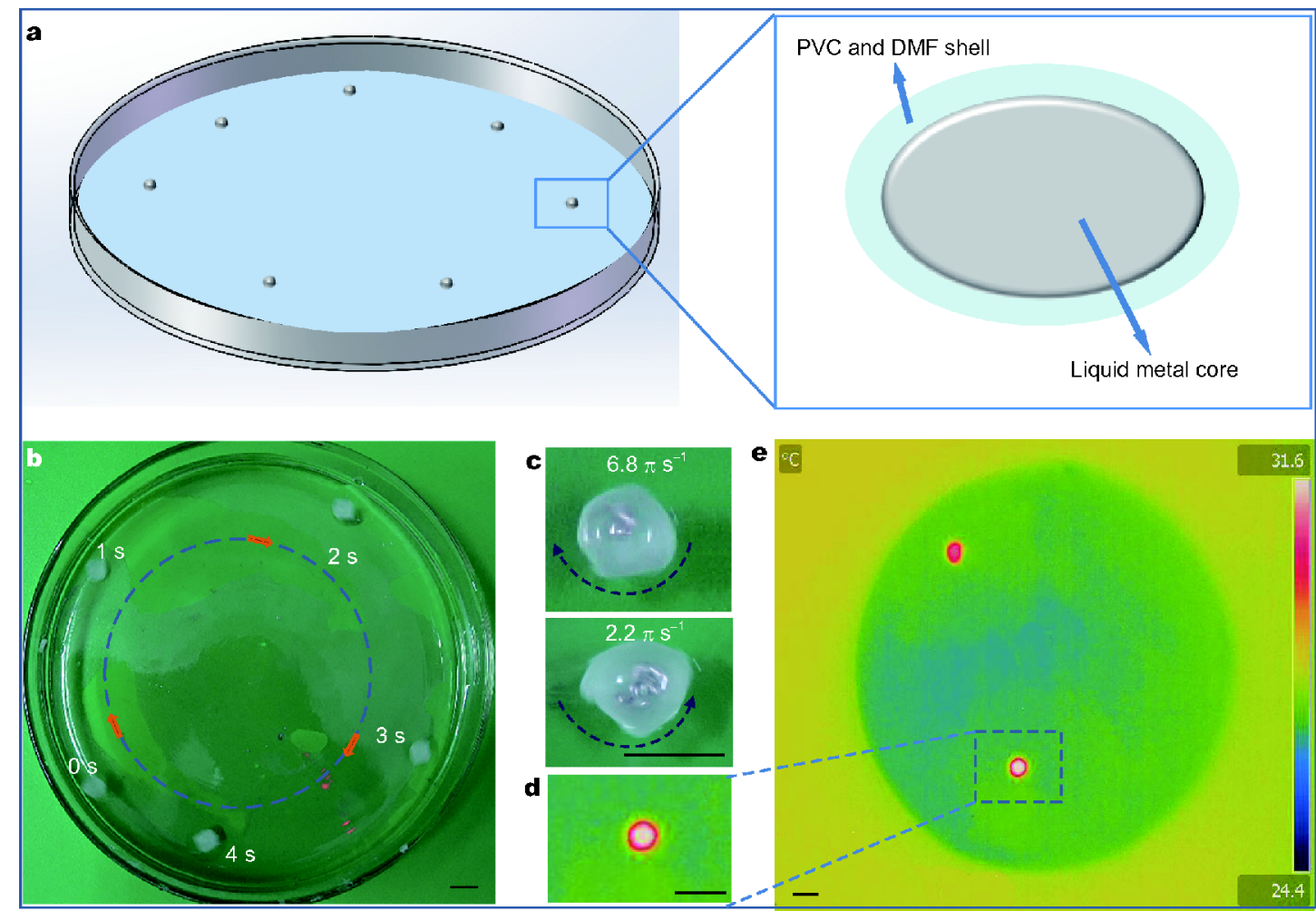

Figure 6 Self-driven motion of PVC-coated LM micro-droplets on water surface. (a) The schematic of the self-driven motion on water surface and the core-shell structure. (b) Self-driven motion on the glass dish. (c) Spin motion of the LM droplets. (d) The close-up view of PVC-coated LM droplets. (e) Infrared image of self-driven motion of PVC-coated LM droplets on water surface. The scale bar is $5 \mathrm{~mm}$. 
moved freely on water surface. More specifically, the behaviors of motion were different for different amounts of LM contained in the PVC shell. Self-driven motion on the glass dish took place when few LM $(0.02 \mathrm{~g})$ microdroplets were carried by the PVC shell (Fig. 6b). With the increase of the weight of LM micro-droplets, spin motions tended to occur. As shown in Fig. 6c, PVC shell with $0.04 \mathrm{~g}$ LM core spun on the water at an angular velocity of $6.8 \pi \mathrm{s}^{-1}$. The angular velocity drops to $2.2 \pi \mathrm{s}^{-1}$ with the increase in the mass of the LM core (Fig. 6c). Spin motion clockwise or anticlockwise mainly originated from asymmetric release of DMF solution and the shift of center of gravity.

Interfacial tension gradients in the surrounding solution were the driving forces for the self-driven motion of PVC-coated LM droplets in the present experiment. The interfacial tension gradient was generated surrounding the PVC-coated LM droplets during the release of the DMF in PVC particles. Further, thermal gradients originating from PVC liquid-solid phase transition also contributed to the generation of interfacial tension gradients. Such synergistic effect could enhance the interfacial tension gradient, generating further stronger driving force. Therefore, this movement will continue until the temperature gradient between the droplets and the environment disappears and the chemical surfactant (DMF) in solid is completely consumed.

To observe the temperature gradient more intuitively, infrared images were taken (Fig. $6 \mathrm{~d}$ and e). The colors reflect the temperature gradients in different regions (the temperature from $24.4-31.6^{\circ} \mathrm{C}$ was recorded in Fig. 6e). The light and red spheres represent the PVC-coated LM micro-particles, which have a higher temperature than the surrounding water. Here, obvious temperature gradients exist, which is the important reason for the interfacial tension gradients, eventually leading to the selfdriven motion. Besides, the infrared experiment also shows that a light yellow area appeared where the droplets have passed (Fig. 6d), which proves the successful release of DMF solvent. The interaction effect of the released DMF supported the self-driven motion of PVCcoated LM droplets and drived it to move along the opposite direction. Therefore, PVC-coated LM micro-droplets can move quickly due to the interfacial tension gradients derived from the release of DMF and temperature difference.

\section{CONCLUSION}

In summary, a practical and efficient method with portable equipment for manufacturing LM micro-droplets is proposed. The size distribution of the produced microdroplets is controlled by tuning the vibration frequency. Moreover, the controllable switching between dispersion and reunion of LM droplets can be realized by adjusting the frequency and amplitude of the vibration. Besides, two potential applications have been presented. Coating with LM micro-droplets retains good optical properties of LM. The self-driven motion of the PVC-coated LM droplets is realized by utilizing the produced LM microdroplets and thus opens up a new strategy for transporting LM droplets. The present work suggests an entirely feasible method for dispersing and utilizing LM droplets, which is of great significance for promoting the development of LM micro-droplet science and technology.

Received 21 June 2018; accepted 17 July 2018; published online 7 August 2018

1 Zhao X, Xu S, Liu J. Surface tension of liquid metal: role, mechanism and application. Front Energy, 2017, 11: 535-567

2 Liu T, Sen P, Kim CJ. Characterization of nontoxic liquid-metal alloy galinstan for applications in microdevices. J Microelectromech Syst, 2012, 21: 443-450

3 Jin C, Zhang J, Li X, et al. Injectable 3-D fabrication of medical electronics at the target biological tissues. Sci Rep, 2013, 3: 3442

4 Ilyas N, Cook A, Tabor CE. Designing liquid metal interfaces to enable next generation flexible and reconfigurable electronics. Adv Mater Interfaces, 2017, 4: 1700141

5 Chen Y, Zhou T, Li Y, et al. Robust fabrication of nonstick, noncorrosive, conductive graphene-coated liquid metal droplets for droplet-based, floating electrodes. Adv Funct Mater, 2018, 28: 1706277

6 Lazarus N, Bedair SS, Kierzewski IM. Ultrafine pitch stencil printing of liquid metal alloys. ACS Appl Mater Interfaces, 2017, 9: $1178-1182$

7 Wang Q, Yu Y, Yang J, et al. Fast fabrication of flexible functional circuits based on liquid metal dual-trans printing. Adv Mater, 2016, 27: 7109-7116

8 Dai D, Zhou Y, Liu J. Liquid metal based thermoelectric generation system for waste heat recovery. Renew Energy, 2011, 36: 35303536

9 Yang XH, Tan SC, He ZZ, et al. Evaluation and optimization of low melting point metal PCM heat sink against ultra-high thermal shock. Appl Thermal Eng, 2017, 119: 34-41

10 Deng Y, Liu J. A liquid metal cooling system for the thermal management of high power LEDs. Int Commun Heat Mass Transfer, 2010, 37: 788-791

11 Wang L, Liu J. Liquid phase 3D printing for quickly manufacturing conductive metal objects with low melting point alloy ink. Sci China Technol Sci, 2014, 57: 1721-1728

12 Yu Y, Liu F, Zhang R, et al. Suspension 3D printing of liquid metal into self-healing hydrogel. Adv Mater Technol, 2017, 2: 1700173

13 Jeon J, Lee JB, Chung SK, et al. On-demand magnetic manipulation of liquid metal in microfluidic channels for electrical switching applications. Lab Chip, 2016, 17: 128-133

14 Jung T, Yang S. Highly stable liquid metal-based pressure sensor 
integrated with a microfluidic channel. Sensors, 2015, 15: 1182311835

15 Gao M, Gui L. A handy liquid metal based electroosmotic flow pump. Lab Chip, 2014, 14: 1866-1872

16 Chechetka SA, Yu Y, Zhen X, et al. Light-driven liquid metal nanotransformers for biomedical theranostics. Nat Commun, 2017, 8: 15432

17 Zhang J, Guo R, Liu J. Self-propelled liquid metal motors steered by a magnetic or electrical field for drug delivery. J Mater Chem B, 2016, 4: 5349-5357

18 Ohira H, Ara K. Development of functional LM by dispersing nano-droplets. Chinese J Biol, 2012, 25: 1039-1042

19 Tang SY, Joshipura ID, Lin Y, et al. Liquid-metal microdroplets formed dynamically with electrical control of size and rate. Adv Mater, 2016, 28: 604-609

20 Fang WQ, He ZZ, Liu J. Electro-hydrodynamic shooting phenomenon of liquid metal stream. Appl Phys Lett, 2014, 105: 134104

21 Tian L, Gao M, Gui L. A microfluidic chip for liquid metal droplet generation and sorting. Micromachines, 2017, 8: 39

22 Tang SY, Ayan B, Nama N, et al. On-chip production of sizecontrollable liquid metal microdroplets using acoustic waves. Small, 2016, 12: 3861-3869

$23 \mathrm{Lu} \mathrm{Y,} \mathrm{Hu} \mathrm{Q,} \mathrm{Lin} \mathrm{Y,} \mathrm{et} \mathrm{al.} \mathrm{Transformable} \mathrm{liquid-metal} \mathrm{nanomedi-}$ cine. Nat Commun, 2015, 6: 10066

24 Tropmann A, Lass N, Paust N, et al. Pneumatic dispensing of nano- to picoliter droplets of liquid metal with the StarJet method for rapid prototyping of metal microstructures. Microfluid Nanofluid, 2012, 12: 75-84

25 Yu Y, Wang Q, Yi L, et al. Channelless fabrication for large-scale preparation of room temperature liquid metal droplets. Adv Eng Mater, 2013, 16: 255-262

26 Hutter T, Bauer WAC, Elliott SR, et al. Formation of spherical and non-spherical eutectic gallium-indium liquid-metal microdroplets in microfluidic channels at room temperature. Adv Funct Mater, 2012, 22: 2624-2631

27 Candelaria SL, Uchaker E, Cao G. Comparison of surface and bulk nitrogen modification in highly porous carbon for enhanced supercapacitors. Sci China Mater, 2015, 58: 521-533

$28 \mathrm{Xu} \mathrm{X}$, Yi D, Wang Z, et al. Greatly enhanced anticorrosion of $\mathrm{Cu}$ by commensurate graphene coating. Adv Mater, 2018, 30: 1702944

29 Zhang Q, Roach DJ, Geng L, et al. Highly stretchable and conductive fibers enabled by liquid metal dip-coating. Smart Mater Struct, 2018, 27: 035019

30 Chen Y, Liu Z, Zhu D, et al. Liquid metal droplets with high elasticity, mobility and mechanical robustness. Mater Horiz, 2017, 4: 591-597

31 Scriven LE, Sternling CV. The marangoni effects. Nature, 1960, 187: $186-188$

32 Tang SY, Khoshmanesh K, Sivan V, et al. Liquid metal enabled pump. Proc Natl Acad Sci USA, 2014, 111: 3304-3309

33 Wang L, Yuan B, Lu J, et al. Self-propelled and long-time transport motion of PVC particles on a water surface. Adv Mater, 2016, 28: 4065-4070

34 Keiser L, Bense H, Colinet P, et al. Marangoni bursting: Evaporation-induced emulsification of binary mixtures on a liquid layer. Phys Rev Lett, 2017, 118: 074504

Acknowledgements This work was partially supported by the Key Project of National Natural Science Foundation of China (91748206), Dean's Research Funding and the Frontier Project of the Chinese Academy of Sciences.

Author contributions Chen S, Wang L and Liu J conceived the idea. Chen $S$ and Wang $L$ carried out all the experiments. All authors participated in the discussion and the preparation of the manuscript. All authors have given approval to the final version of the manuscript.

Conflict of interest The authors declare that they have no conflict of interest.

Supplementary information Supporting data are available in the online version of the paper. 

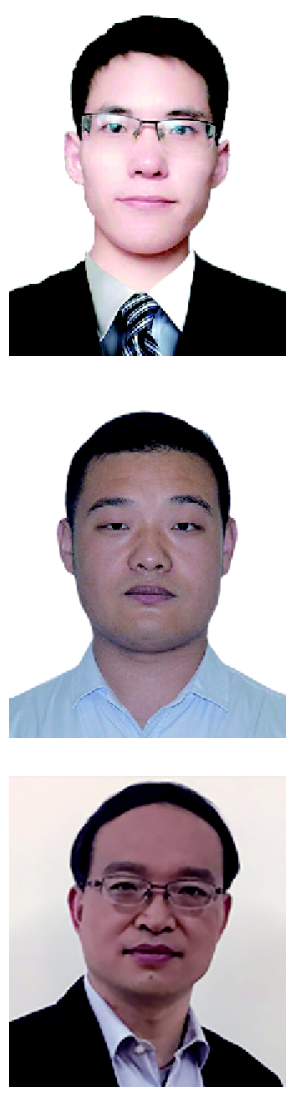

Sen Chen received his bachelor's degree from Huazhong University of Science and Technology in 2016. Now he is pursuing his doctor's degree at the Technical Institute of Physics and Chemistry of the Chinese Academy of Sciences. His research interests focus on the properties and applications of room temperature liquid metals.
Lei Wang received his $\mathrm{PhD}$ degree from Beihang University in 2015, under the supervision of Prof. Yong-Mei Zheng. In 2017, he joined the Technical Institute of Physics and Chemistry, Chinese Academy Sciences. Now, he is an assistant researcher at the Technical Institute of Physics and Chemistry. His research interests are focused on the combination of liquid metal and bionic materials.

Jing Liu is a jointly appointed Professor of Tsinghua University (THU) and Technical Institute of Physics and Chemistry, Chinese Academy of Sciences. He received his double BSc degrees in Engineering and Physics, and PhD, all from THU and performed visiting research at Purdue University and MIT, USA. Dr. Liu works at the interdisciplinary areas among liquid metal, biomedical engineering, and thermal science and is an author of nine popular books. His research on liquid metal chip cooling, printed electronics, biomaterials, and soft machines initiated many game-changing technologies. Dr. Liu pioneered a group of very fundamental discoveries on liquid metals which were frequently featured over the world.

\section{液态金属液滴的可控分散和融合}

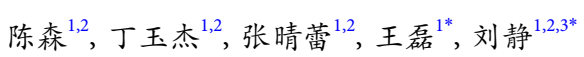

摘要 结合了液态金属和微尺度的优点, 液态金属微液滴由于其优异的性能近年来引起了人们的高度关注, 并已被广泛应用于微流体、 载药纳米系统和微纳米机器中. 然而, 由于液态金属巨大的表面张力, 液态金属微纳米液滴的高效简易制备仍然存在困难, 特别是其分散 和融合的可控且智能切换仍有待实现. 基于此, 本文提出了一种高效制备液体金属微纳米液滴的新方法, 并实现了液态金属液滴的可控分 散和融合. 浸入 $N, N$ 二甲基甲酰胺(DMF)和聚氯乙烯(PVC)混合溶液中的液态金属, 通过振动可以产生微纳米级的液态金属微液滴. 实验 结果表明, 控制振动的频率可以调节液态金属微液滴的尺寸分布. 更令人惊喜的是, 通过调整振动的频率和振幅, 可以实现液态金属液滴 的分散和融合的智能切换, 这在可变形柔性机器等领域有着重要应用. 此外, 文章还对利用这种方法制作的液态金属微液滴涂层的光学性 质进行了评估, 以证明其具有更多潜在的应用. 最后, 文章演示了利用本方法所制备的PVC包覆的液态金属微液滴可以在水面实现自驱动 运动, 这对未来液态金属微液滴的运输具有重要的意义. 本文提出的这种完全可行的液态金属微液滴分散融合及其实现的方法对于液态 金属微液滴科学和技术的发展具有显著的促进作用. 